\title{
Importance of surface carboxyl groups on biofouling development and control for thin film composite (TFC) polyamide membranes
}

Jiayu Tian ${ }^{\mathrm{a}, \mathrm{c}}$, Xiujuan Hao ${ }^{\mathrm{b}, \mathrm{c}, *}$, Xinglin $\mathrm{Lu}^{\mathrm{d}}$, Shanshan Gao ${ }^{\mathrm{a}}$, Ruijun Zhang ${ }^{\mathrm{a}}$, Songxue Wang ${ }^{\mathrm{e}}$, Fuyi Cuif

${ }^{a}$ School of Civil and Transportation Engineering, Hebei University of Technology, Tianjin, 300401, China.

${ }^{b}$ School of Civil Engineering, Inner Mongolia University of Technology, Huhhot, 010051, China.

${ }^{c}$ State Key Laboratory of Urban Water Resource and Environment, Harbin Institute of Technology, Harbin, 150090, China

${ }^{d}$ Department of Chemical and Environmental Engineering, Yale University, New Haven, Connecticut, 06520-8286, United States.

e College of Environmental and Municipal Engineering, Qingdao University of Technology, Qingdao, 266033, China.

${ }^{f}$ School of Environment and Ecology, Chongqing University, Chongqing, 400044, China.

*Email: hxj_hit@163.com. 
Total number of pages (including the cover page): 5

Number of figures: 4

Number of tables: 1

Page S3 S1. Simulated wastewater composition

Page S3 S2. Characterization of the CCTS-modified membranes 


\section{S1. Simulated wastewater composition.}

Table S1 Chemical composition of the simulated wastewater in biofouling experiment $(\mathrm{pH}=7.5)$

\begin{tabular}{cc}
\hline Composition & Concentration (mM) \\
\hline $\mathrm{NaCl}$ & 8 \\
$\mathrm{NH}_{4} \mathrm{Cl}$ & 0.4 \\
$\mathrm{KHPO}_{4}$ & 0.2 \\
$\mathrm{NaHCO}_{3}$ & 0.5 \\
Sodium citrate & 0.6 \\
\hline
\end{tabular}

\section{S2. Characterization of the CCTS-modified membranes}

Membrane performance was evaluated in FO mode, using DI water as feed solution, $0.5-2.5 \mathrm{M} \mathrm{NaCl}$ as draw solutions, crossflow velocity of $8.5 \mathrm{~cm} / \mathrm{s}$, and temperature of $23{ }^{\circ} \mathrm{C}$.
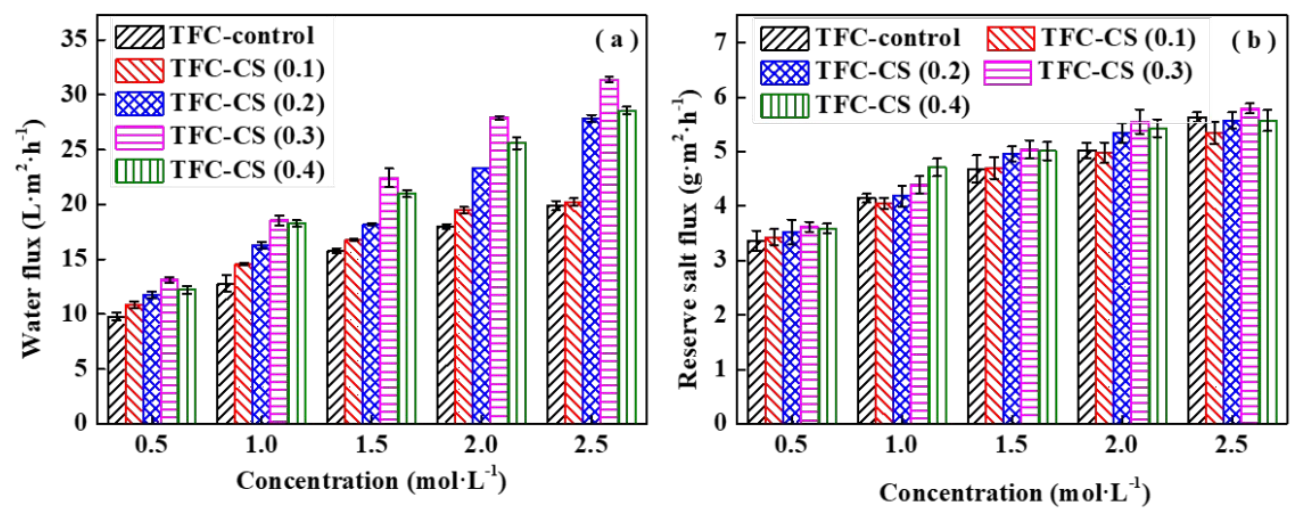

Figure S1. Water flux (a) and reverse salt flux (b) of the TFC-control membrane and the CCTS-modified membranes in FO mode.

SEM results (Figure S1a-d) show that the TFC-control membrane surface includes some larger leaf-like structures. In contrast, the TFC-CS modified membranes with CCTS loading of $0.1 \sim 0.3$ wt.\% showed more nodular features at higher CCTS loading, which was responsible for the reduced surface roughness (Figure S2). 
(a)

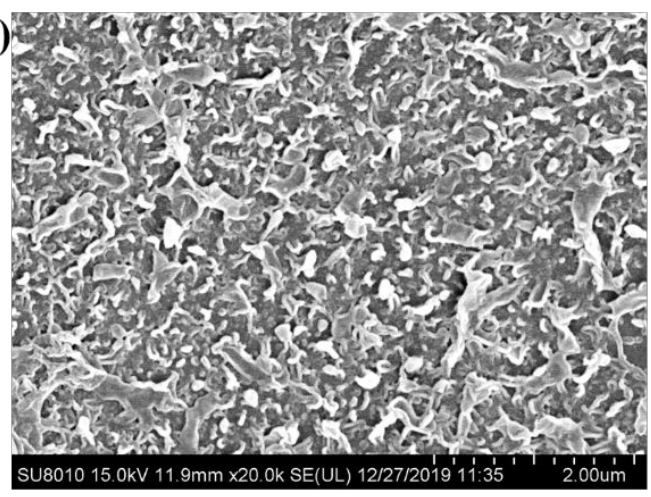

(c)

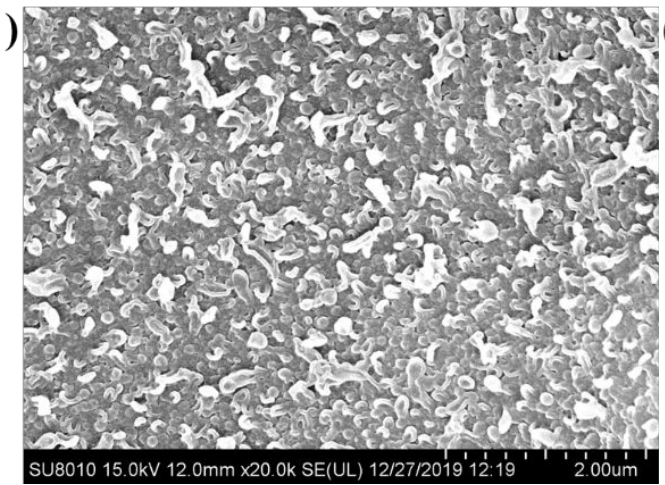

(b)

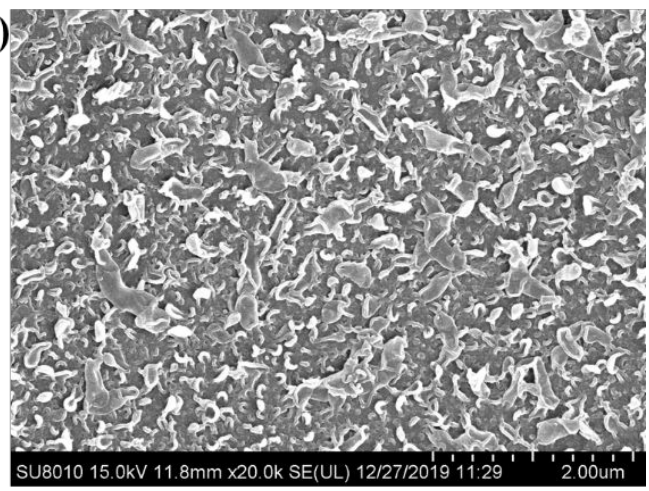

d)

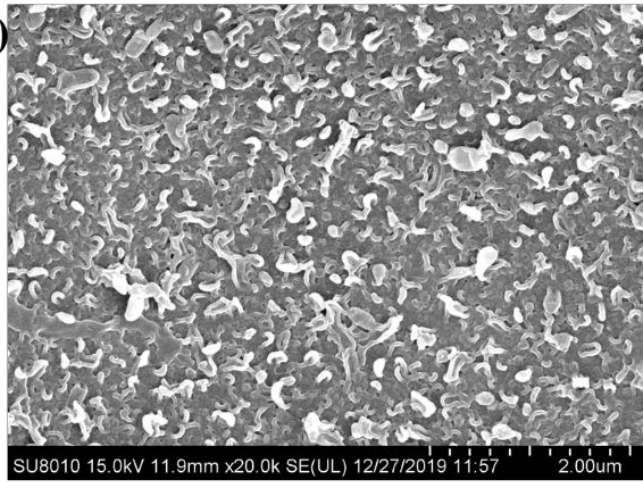

Figure S2. SEM images of the polyamide active layer of (a) TFC-control, (b) TFC-CS (0.1), (c)

TFC-CS (0.2) and (d) TFC-CS (0.3).

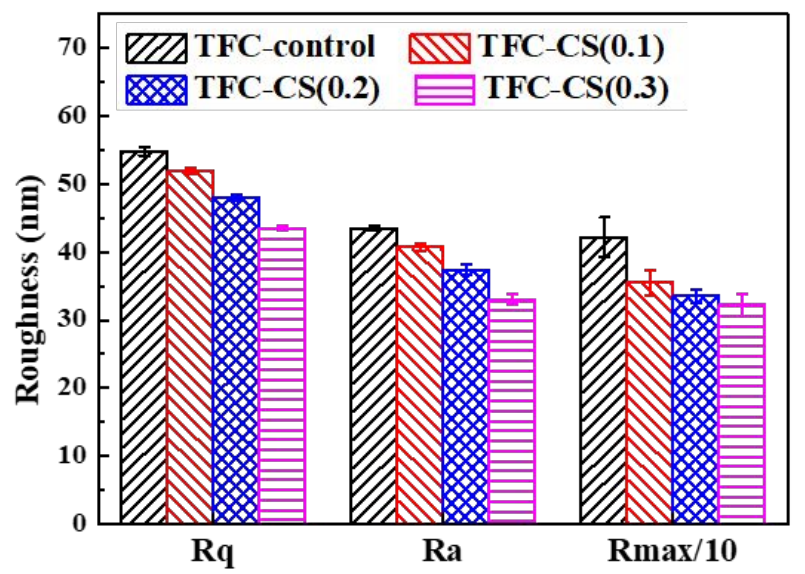

Figure S3. AFM images of the polyamide active layer of TFC-control and TFC-CS membranes.

S3. Fouling reversibility. 


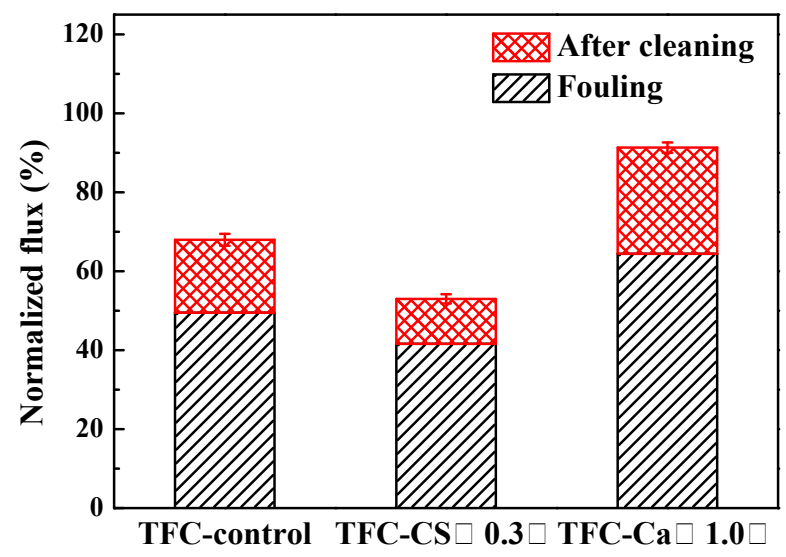

Figure S4 Fouling reversibility was evaluated by cleaning the fouling membranes with DI water under crossflow conditions. Error bars are based on the range of the two repeated tests. 